\title{
Chrysoeriol ameliorates COX-2 expression through NF-кB, AP-1 and MAPK regulation via the TLR4/MyD88 signaling pathway in LPS-stimulated murine macrophages
}

\author{
HYUN-SEO YOON ${ }^{1,2}$ and CHUNG MU PARK ${ }^{2,3}$ \\ ${ }^{1}$ Department of Dental Hygiene; ${ }^{2}$ The Research Institute for Health Functional Materials; \\ ${ }^{3}$ Department of Clinical Laboratory Science, Dong-Eui University, Busanjin-gu, Busan 47340, Republic of Korea
}

Received November 6, 2020; Accepted February 16, 2021

DOI: $10.3892 /$ etm.2021.10150

\begin{abstract}
Chrysoeriol is a flavonoid that has diverse biological properties, including antioxidation, anti-inflammation, chemoprevention and immunomodulation. Despite its reported anti-inflammatory activity, the exact underlying molecular mechanism has not yet been elucidated. In the current study, the anti-inflammatory mechanism of chrysoeriol involving lipopolysaccharide (LPS)-induced cyclooxygenase-2 (COX-2) and its upstream signaling molecules was investigated in RAW 264.7 cells. The mechanism was evaluated via ELISA and western blotting assays. Chrysoeriol significantly inhibited LPS-induced prostaglandin $\mathrm{E}_{2}\left(\mathrm{PGE}_{2}\right)$ production and COX-2 expression without cytotoxicity. Activated transcription factors that further induced the inflammation response, including nuclear factor $(\mathrm{NF})-\kappa \mathrm{B}$ and activator protein-1 (AP-1), were significantly attenuated by chrysoeriol treatment. Furthermore, LPS-induced phosphorylation levels of phosphoinositide-3-kinase (PI3K)/Akt and mitogen-activated protein kinase (MAPK) were abolished by chrysoeriol treatment, which was confirmed by selective inhibitors. Additionally, chrysoeriol significantly inhibited the LPS-induced activation of adaptor molecules in RAW 264.7 cells, including toll-like receptor 4 (TLR4) and myeloid differentiation primary response 88 . Therefore, the results suggested that chrysoeriol ameliorates TLR4-mediated inflammatory responses by inhibiting NF- $\mathrm{NB}$ and AP-1 activation as well as suppressing PI3K/Akt and MAPK phosphorylation in LPS-stimulated RAW 264.7 cells.
\end{abstract}

Correspondence to: Dr Chung Mu Park, Department of Clinical Laboratory Science, Dong-Eui University, 176 Eomgwangro, Busanjin-gu, Busan 47340, Republic of Korea

E-mail: cmpark@deu.ac.kr

Key words: chrysoeriol, cyclooxygenase-2, nuclear factor- $\kappa \mathrm{B}$, activator protein-1, phosphoinositide 3-kinase, mitogen-activated protein kinase, toll like receptor 4 , myeloid differentiation primary response 88

\section{Introduction}

Inflammation is a protective mechanism that can counteract diverse biological stimuli; however, chronic inflammation can play significant roles in the progress of various disorders such as cancer, chronic respiratory diseases, heart disorders, and diabetes $(1,2)$. Excessively generated inflammatory mediators, including nitric oxide $(\mathrm{NO})$, prostaglandin $\mathrm{E}_{2}\left(\mathrm{PGE}_{2}\right)$, and inflammatory cytokines, are involved in the development of inflammatory responses (3). Among them, $\mathrm{PGE}_{2}$ can be generated by the rate-limiting enzyme cyclooxygenase-2 (COX-2) from arachidonic acid, which is usually elevated in response to inflammatory stimuli such as chemical injury and tumor promoters (4). Thus, the arachidonic acid pathway has been regarded as one of the hallmarks of chronic inflammation. Moreover, the increased production of $\mathrm{PGE}_{2}$ induced by the upregulated expression of COX-2 can be observed in various premalignant and malignant tissues (5). It has also been reported that the upregulation of COX-2 can lead to inhibited apoptosis and accelerated malignant cell invasion (6), which are reversed by non-steroidal anti-inflammatory agents. Therefore, inhibitors of COX-2 expression may be considered as promising therapeutics acting as preventive agents against cancer and chronic inflammation (7).

Chrysoeriol is a flavone that is found in Flos Lonicerae (Lonicera japonica flowers), Tanacetum vulgare, Artemisia arborescens, Salix matsudana leaves, Aspalathus linearis, and Coronopus didymus (8-12). Furthermore, chrysoeriol has numerous pharmacological properties, such as anti-inflammation, antioxidation, relaxing smooth muscle, reducing obesity, and regulating the immune system (8-14). While a previous study has revealed the anti-inflammatory activity of chrysoeriol in the RAW 264.7 cell line (13), its exact mechanism involving COX-2 inhibition is not fully understood. Therefore, the present study aimed to investigate the molecular mechanisms of chrysoeriol on lipopolysaccharide (LPS)-induced inflammation in the RAW 264.7 cell line.

\section{Materials and methods}

Reagents. Dulbecco's modified Eagle's medium (DMEM) and fetal bovine serum (FBS) were obtained from Cytiva. 
Chrysoeriol was purchased from ChromaDex (analytical grade verified by HPLC or GC analysis; cat. no. ASB-00003630-005) and dissolved in dimethyl sulfoxide (DMSO; cat. no. D8418; Sigma-Aldrich). LY294002 (purity: $\geq 98 \%$; cat. no. L9908), SP600125 (purity: $\geq 98 \%$; cat. no. S5567) and SB202190 (purity: $\geq 98 \%$; cat. no. S7067) were obtained from Sigma-Aldrich; Merck KGaA which was applied as selective inhibitor for phosphoinositde 3-kinase (PI3K)/Akt, c-Jun $\mathrm{NH}_{2}$-terminal kinase (JNK) and p38, respectively.

Cell culture. RAW 264.7 cell line was purchased from American Type Culture Collection (cat. no. TIB-71) and was cultured in DMEM supplemented with $10 \%$ FBS and $2 \mathrm{mM}$ L-glutamine (Hyclone; Cytiva). Cells were seeded in $100 \mathrm{~mm}$ dishes $\left(5 \times 10^{6}\right.$ cells/dish) and preincubated with indicated concentrations of chrysoeriol for $2 \mathrm{~h}$ and then incubated with LPS ( $1 \mu \mathrm{g} / \mathrm{ml}$; cat. no. L4516) for $18 \mathrm{~h}$ to analyze the expression levels of inflammatory mediators (15). To identify transcription factors and upstream signaling molecules, cells were treated with the indicated concentrations of chrysoeriol for $2 \mathrm{~h}$ along with LPS $(1 \mu \mathrm{g} / \mathrm{ml})$ (16). In addition, $20 \mu \mathrm{M}$ of selective inhibitors for PI3K/Akt and mitogen-activated protein kinases (MAPKs), as well as chrysoeriol, were pre-incubated for $2 \mathrm{~h}$, and then incubated with LPS $(1 \mu \mathrm{g} / \mathrm{ml})$ for $18 \mathrm{~h}$ to investigate which signaling molecules are related to the anti-inflammatory responses (17).

Cell viability. Cell viability was determined by the CellTiter 96 Aqueous one solution cell proliferation assay (cat. no. G3582; Promega). RAW 264.7 cells were seeded in 24-well plate $\left(4 \times 10^{5}\right.$ cells/well) and incubated with or without various concentrations of chrysoeriol for $24 \mathrm{~h}$ (15). Then, $50 \mu \mathrm{l}$ of MTS solution was added to $950 \mu \mathrm{l}$ of DMEM and incubated for $1 \mathrm{~h}$ at $37^{\circ} \mathrm{C}$, then the absorbance was measured at $490 \mathrm{~nm}$ with an xMark Microplate Absorbance Spectrophotometer (Bio-Rad Laboratories, Inc.).

$P G E_{2}$ determination. $\mathrm{PGE}_{2}$ concentration was measured using an enzyme-linked immunosorbent assay (ELISA) kit (cat. no. 500141; Cayman Chemical), following the manufacturer's instructions. Briefly, RAW 264.7 cells were seeded in a 24-well plate and preincubated with indicated concentrations of chrysoeriol for $2 \mathrm{~h}$ and then incubated with LPS $(1 \mu \mathrm{g} / \mathrm{ml})$ for $18 \mathrm{~h}$ to analyze the concentration of $\mathrm{PGE}_{2}$. Briefly, $50 \mu \mathrm{l}$ of supernatant of culture medium and the equal volume of $\mathrm{PGE}_{2}$ tracer were mixed in the $\mathrm{PGE}_{2}$ ELISA plate and incubated for $18 \mathrm{~h}$ at $4^{\circ} \mathrm{C}$. The wells were rinsed for 5 times with wash buffer. Then, $200 \mu 1$ of Ellman's reagent was added to the well and incubated in the dark in order to develop. The absorbance was measured at $405 \mathrm{~nm}$ with an xMark Microplate Absorbance Spectrophotometer (Bio-Rad Laboratories, Inc.) (18).

Western blot analysis. Antibodies for COX-2 (1:1,000; cat. no. 12282), phospho-p65 (1:1,000; cat. no. 3033), p65 (1:1,000; cat. no. 8242), phospho-c-jun (1:1,000; cat. no. 3270), c-jun (1:1,000; cat. no. 9165), phospho-Akt (1:1,000; cat. no. 4060), Akt (1:1,000; cat. no. 4691), phospho-extracellular signal-regulated kinase (ERK; 1:1,000; cat. no. 8544), ERK (1:1,000; cat. no. 4695), phospho-JNK (1:1,000; cat. no. 4668), JNK (1:1,000; cat. no. 9252), phospho-p38 (1:1,000; cat. no. 4511), p38 (1:1,000; cat. no. 8690) and actin (1:1,000; cat. no. 4970) as well as the horseradish peroxidase (HRP)-conjugated anti-rabbit IgG (1:1,000; cat. no. 7074) were purchased from Cell Signaling Technology. Antibodies against Toll-like receptor 4 (TLR4; 1:500; cat. no. ab13356) and myeloid differentiation primary response 88 (MyD88; 1:500; cat. no. ab2064) were obtained from Abcam. RAW 264.7 cells were incubated with indicated concentrations of chrysoeriol for $2 \mathrm{~h}$ and then treated with $1 \mu \mathrm{g} / \mathrm{ml}$ LPS for $18 \mathrm{~h}$. Cells were washed with PBS and harvested using M-PER ${ }^{\mathrm{TM}}$ mammalian protein extraction reagent (cat. no. 78051; Thermo Fisher Scientific, Inc.) for $10 \mathrm{~min}$ at room temperature. Cell lysis buffer was centrifuged at 13,000 x $\mathrm{g}$ for $10 \mathrm{~min}$ and the protein concentration was determined by Bradford assay. Then, $50 \mu \mathrm{g}$ protein samples were separated on a $10 \%$ SDS-PAGE gel and transferred to a PVDF membrane (Bio-Rad Laboratories, Inc.). After transfer, the membrane was blocked with 5\% skim milk for $2 \mathrm{~h}$ at room temperature. Then, each diluted primary antibody was incubated with the membranes for overnight at $4^{\circ} \mathrm{C}$. After washing the membranes with PBST, they were incubated with horseradish peroxidase-conjugated anti-rabbit IgG secondary antibody for $2 \mathrm{~h}$ at room temperature. The membrane was developed with ECL substrate solution (Santa Cruz Biotechnology, Inc.) and western blotting data were quantified using a Gel Doc EQ System (Bio-Rad Laboratories, Inc.).

Statistical analysis. Data are presented as the mean \pm SD. Statistical analyses were performed using SPSS version 25.0 (IBM Corp.). One-way ANOVA with Tukey's multiple comparison test was used to analyze the difference between each group. $\mathrm{P}<0.05$ was considered to indicate a statistically significant difference.

\section{Results}

Chrysoeriol inhibits $P G E_{2}$ secretion and $C O X-2$ expression in LPS-treated RAW 264.7 cells. The anti-inflammatory effect of chrysoeriol was investigated in LPS-stimulated RAW 264.7 cells. As shown in Fig. 1A, LPS-induced $\mathrm{PGE}_{2}$ production was significantly mitigated by chrysoeriol treatment in a dose-dependent manner, without causing cytotoxicity (Fig. S1). Moreover, the corresponding enzyme of $\mathrm{PGE}_{2}$ formation, COX-2, was also significantly attenuated by chrysoeriol treatment (Fig. 1B).

Chrysoeriol suppresses nuclear factor $(N F)-\kappa B$ and activator protein (AP)-1 activation in LPS-treated RAW 264.7 cells. Western blot analysis was applied in order to analyze the activated status of NF- $\mathrm{B}$ and AP-1, and phosphorylation of each subunit of both transcription factors p65 and c-jun was significantly inhibited by chrysoeriol treatment in a dose-dependent manner (Fig. 2). Therefore, chrysoeriol treatment ameliorated LPS-induced inflammatory circumstances in RAW 264.7 cells.

Chrysoeriol inhibits PI3K and p38 phosphorylation levels via TLR4/MyD88 inactivation in LPS-treated RAW 264.7 cells. To identify the upstream signaling molecules that can regulate NF- $\kappa \mathrm{B}$ and AP-1 activation, the phosphorylation of PI3K and 

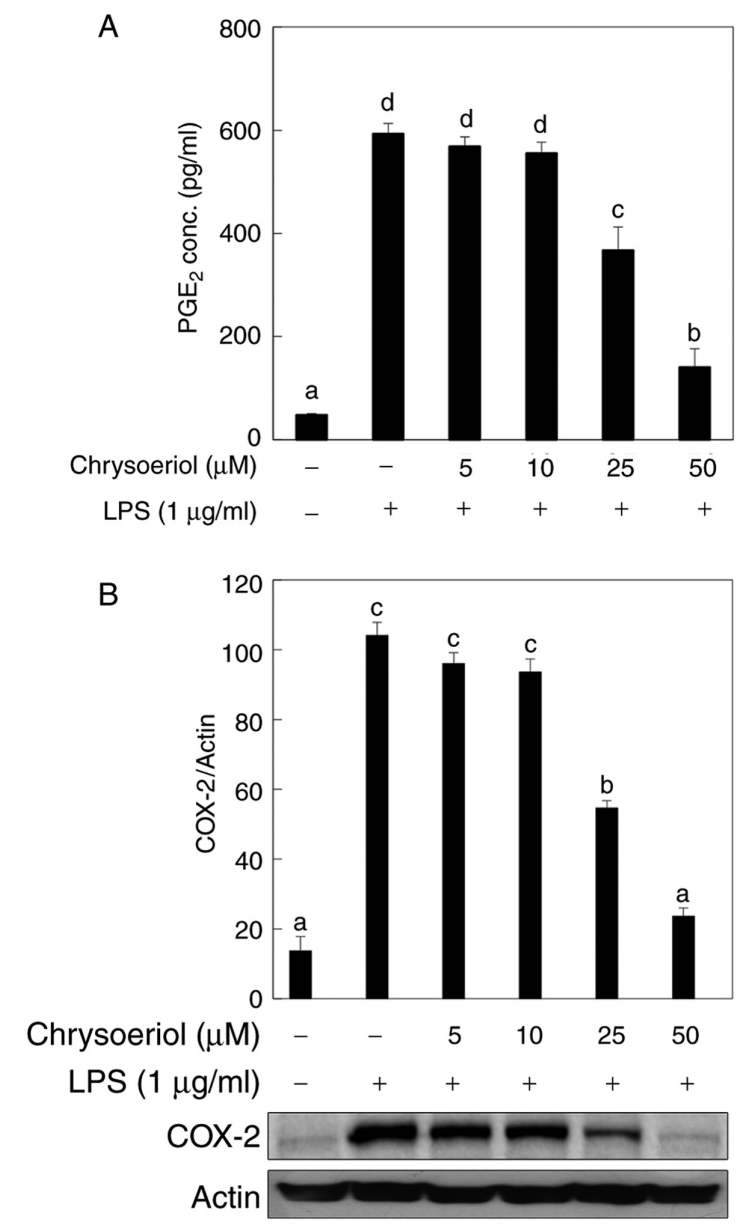

Figure 1. Chrysoeriol inhibited $\mathrm{PGE}_{2}$ production and COX-2 expression in LPS-stimulated RAW 264.7 cells. Cells were pre-incubated with or without the indicated concentrations of chrysoeriol for $2 \mathrm{~h}$, then incubated with LPS $(1 \mu \mathrm{g} / \mathrm{ml})$ for $18 \mathrm{~h}$ at $37^{\circ} \mathrm{C}$ in a humidified atmosphere containing $5 \% \mathrm{CO}_{2}$. (A) $\mathrm{PGE}_{2}$ production was analyzed by an ELISA assay. (B) Protein expression level of COX-2 was assessed following chrysoeriol treatment. The relative induction of COX-2 expression was quantified by densitometry and actin was used as an internal control. Data are presented as the mean \pm SD of triplicate experiments. Values sharing the same superscript letter were not significantly different at $\mathrm{P}<0.05 . \mathrm{PGE}_{2}$, prostaglandin $\mathrm{E}_{2}$; COX-2, cyclooxygenase-2; LPS, lipopolysaccharide.

MAPK was measured by western blot analysis. Chrysoeriol significantly inhibited Akt and p38 phosphorylation, while slightly affecting JNK activation (Fig. 3A). Furthermore, ERK was not affected by chrysoeriol treatment in RAW 264.7 cells.

A selective inhibitor of each signaling molecule was used to assess the role of PI3K and MAPK signaling molecules in LPS-stimulated inflammatory cascades. LY294002 and SB202190, selective inhibitors of PI3K and p38, respectively, significantly inhibited COX-2 expression, but SP600125 did not affect COX-2 expression in LPS-stimulated RAW 264.7 cells (Fig. 3B). Moreover, COX-2 expression was most highly inhibited when LY294002, SB202190, and SP600125 treatments were applied together in RAW 264.7 cells.

In addition, the present study also investigated the effect of chrysoeriol on TLR4 and MyD88, based on their roles as adaptor molecules in the development of NF- $\kappa \mathrm{B}, \mathrm{AP}-1$, PI3K, and MAPKs $(14,15)$ in LPS-stimulated RAW 264.7 cells. It was indicated that chrysoeriol mitigated the activation of TLR4 and MyD88 in a dose-dependent manner, in

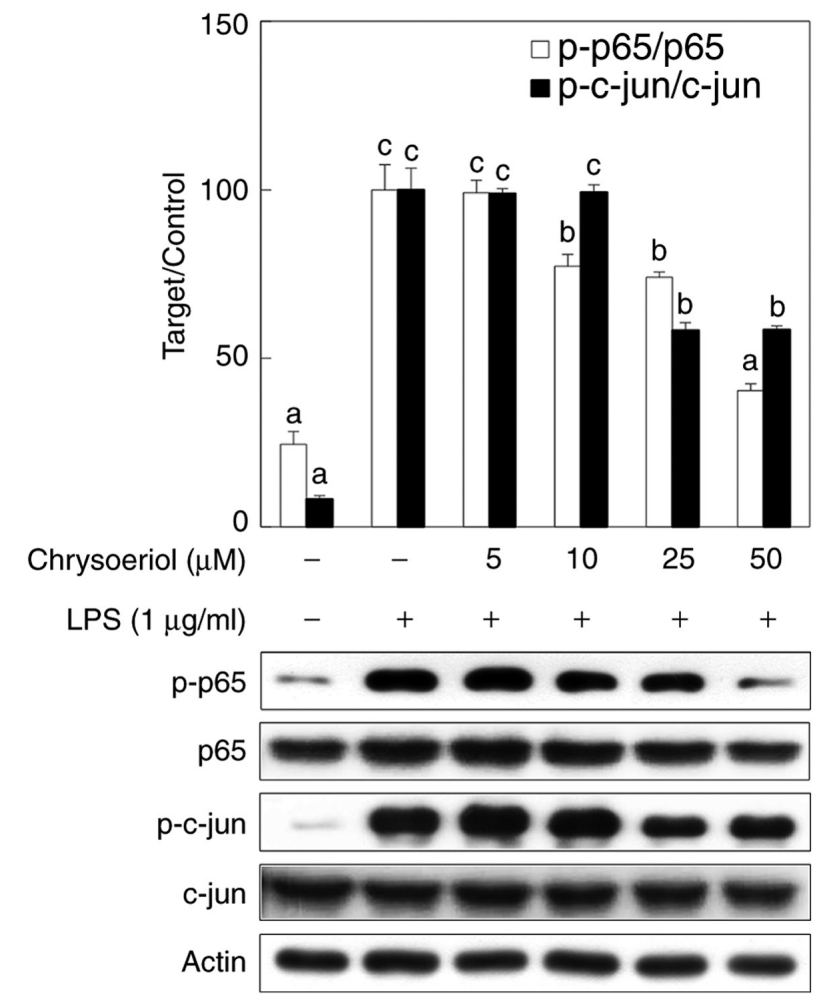

Figure 2. Chrysoeriol inhibited the phosphorylation of transcription factors, NF- $\kappa$ B and AP-1, in LPS-stimulated RAW 264.7 cells. Cells were incubated with or without LPS $(1 \mu \mathrm{g} / \mathrm{ml})$ and with the indicated concentrations of chrysoeriol for $2 \mathrm{~h}$ at $37^{\circ} \mathrm{C}$ in a humidified atmosphere containing $5 \% \mathrm{CO}_{2}$. Chrysoeriol treatment inhibited the phosphorylation of NF- $\kappa$ B and AP-1 subunits (p65 and c-jun) in LPS-stimulated RAW 264.7 cells. P65 and c-jun phosphorylation was quantified by densitometry and unphosphorylated forms of each transcription factor were used as an internal control. Data are presented as the mean \pm SD of triplicate experiments. Values sharing the same superscript letter were not significantly different at $\mathrm{P}<0.05$. NF- $\kappa \mathrm{B}$, nuclear factor- $\kappa \mathrm{B}$; AP-1, activator protein-1; LPS, lipopoly saccharide; p, phosphorylated.

accordance with the inhibited NF- $\mathrm{B}, \mathrm{AP}-1, \mathrm{PI} 3 \mathrm{~K}$, and $\mathrm{p} 38$ MAPK expression levels, in LPS-stimulated RAW 264.7 cells (Fig. 4). Collectively, these results suggested that inhibited PI3K and p38 MAPK phosphorylation levels via TLR4/MyD88 mitigation by chrysoeriol treatment may contribute to reducing LPS-induced NF- $\kappa$ B and AP-1 activation, resulting in reduced $\mathrm{COX}-2$ expression and $\mathrm{PGE}_{2}$ production in RAW 264.7 cells.

\section{Discussion}

After the discovery of COX-2 in 1991, a variety of pharmaceutical candidates were tested to identify their potential uses as selective inhibitors of COX-2 and PG in inflammatory lesions (19). A large number of plant-derived compounds have been examined as candidates as a COX-2 selective inhibitor. Among them, chrysoeriol, which is a flavonoid, has numerous pharmaceutical properties, including anti-inflammatory, antioxidative, and anticarcinogenic activities $(8-13,20)$. It has been reported that the anti-inflammatory activity of chrysoeriol may occur via the inhibition of NO production caused by AP-1 blockage in RAW 264.7 cells (13). However, to the best of our knowledge, there are no previous studies analyzing chrysoeriol 
A
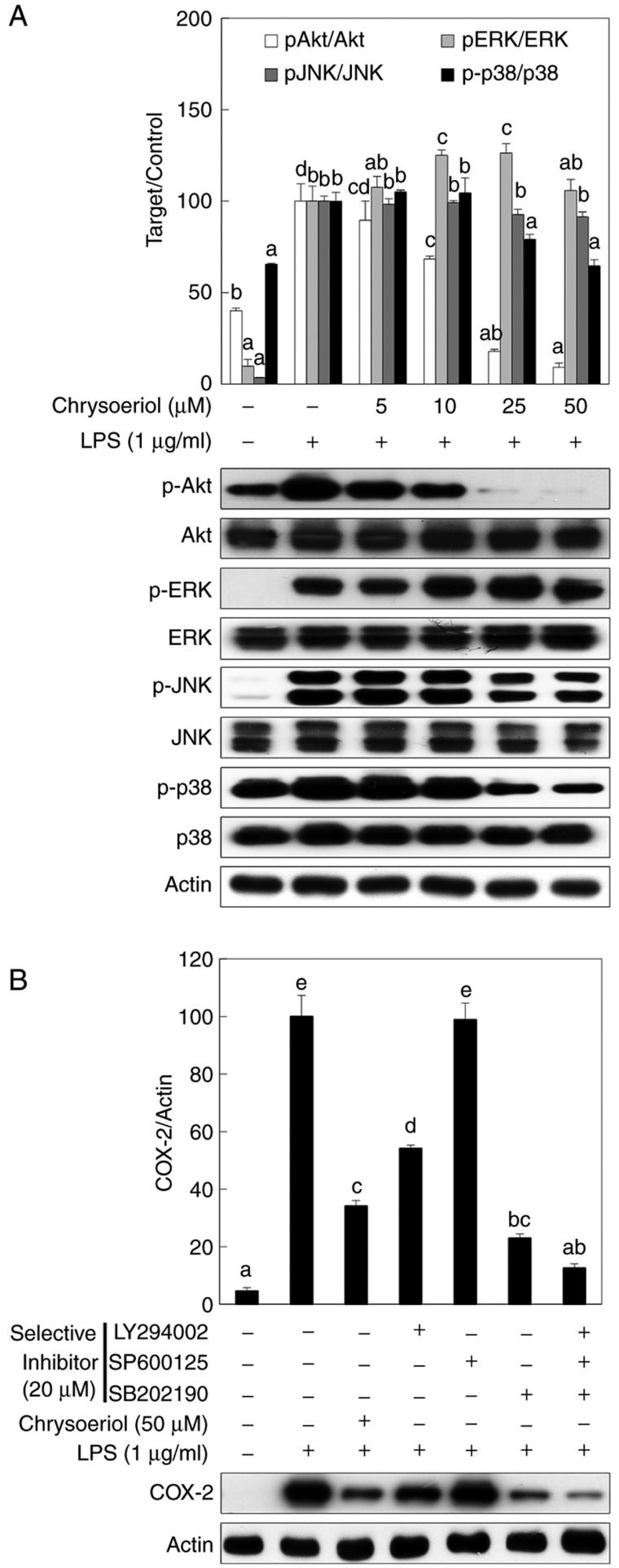

Figure 3. Chrysoeriol inhibited the phosphorylation of Akt and p38, which was confirmed by selective inhibitors in LPS-stimulated RAW 264.7 cells. Cells were incubated with or without LPS $(1 \mu \mathrm{g} / \mathrm{ml})$ and with the indicated concentrations of chrysoeriol for $2 \mathrm{~h}$ at $37^{\circ} \mathrm{C}$ in a humidified atmosphere containing $5 \% \mathrm{CO}_{2}$. (A) Protein expression levels of p-Akt, p-ERK, p-JNK, and p-p38 were assessed following chrysoeriol treatment. Unphosphorylated forms of signaling molecules and actin were used as internal controls. Akt, ERK, JNK and p38 phosphorylation was quantified by densitometry and unphosphorylated forms of each signaling molecule were used as an internal control. (B) A selective inhibitor of each signaling molecule was applied to RAW 264.7 cells. The relative inhibition of COX-2 was quantified by densitometry and actin was used as an internal control. Data are presented as the mean $\pm \mathrm{SD}$ of triplicate experiments. Values sharing the same superscript letter were not significantly different at $\mathrm{P}<0.05$. LPS, lipopolysaccharide; p, phosphorylated; ERK, extracellular signal-regulated kinase; JNK, c-Jun $\mathrm{NH}_{2}$-terminal kinase; COX-2, cyclooxygenase-2.
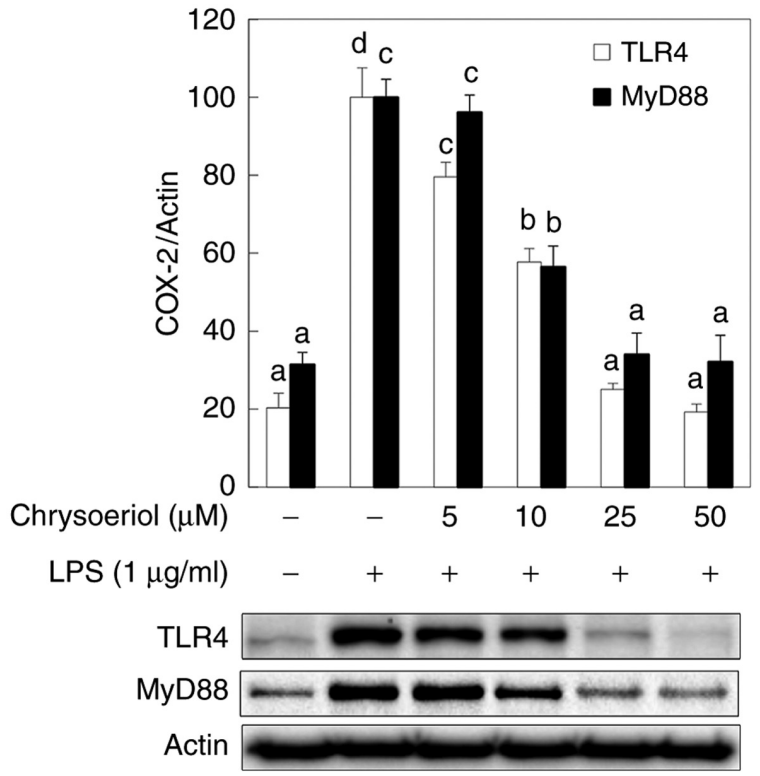

Figure 4. Chrysoeriol inhibited the activation of each adaptor molecule (TLR4 and MyD88) in LPS stimulated RAW 264.7 cells. Cells were incubated with or without LPS $(1 \mu \mathrm{g} / \mathrm{ml})$ and with the indicated concentrations of chrysoeriol for $2 \mathrm{~h}$ at $37^{\circ} \mathrm{C}$ in a humidified atmosphere containing 5\% CO $\mathrm{CO}_{2}$ TLR4 and MyD88 protein expressions was quantified via densitometry and actin was used as an internal control. Data are presented as the mean \pm SD of triplicate experiments. Values sharing the same superscript letter were not significantly different at $\mathrm{P}<0.05$. TLR4, toll like receptor 4; MyD88, myeloid differentiation primary response 88 ; LPS, lipopolysaccharide; COX-2, cyclooxygenase-2.

as a COX-2 inhibitor and identifying its underlying mechanism in LPS-induced inflammatory responses. Therefore, the present study aimed to investigate the anti-inflammatory mechanism of chrysoeriol, focused on COX-2 regulation, in LPS-stimulated murine macrophage cells.

Inflammation is a type of defense mechanism occurring in the immune system against tissue injuries, infections, or toxic materials $(21,22)$. Several pathophysiological disorders, such as cancer, arthritis, cardiovascular disease, atherosclerosis, and neurodegenerative diseases, may be caused by prolonged inflammatory responses when acute inflammation cannot be controlled in the early-stage $(23,24)$. Among various immune cells, macrophages play important roles in host defense mechanisms via the regulation of $\mathrm{NO}$ and $\mathrm{PGE}_{2}$, as well as pro-inflammatory cytokines including tumor necrosis factor (TNF)- $\alpha$, interleukin (IL)-6, and IL-1 $\beta$ (25). LPS, found in the outer membrane of some types of Gram-negative bacteria, activates immune responses by interacting with TLR4 associated with CD14, which induces phosphorylation of MAPKs and subsequently initiates the stimulation of transcription

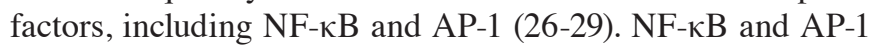
are critical transcription factors that regulate the expression of inflammatory mediators such as iNOS and COX-2. P65 and c-jun, each respective subunits of NF- $\mathrm{kB}$ and AP-1, are phosphorylated and changed to active forms when inflammatory stimuli reach the cells. Then, these subunits translocate into the nucleus and bind to the promoter regions of inflammatory mediators (30). Therefore, the downregulation of inflammatory mediators as well as inflammatory signaling molecules is one of the major targets for ameliorating inflammation and its associated various disorders. In RAW 264.7 cells, LPS induced 
$\mathrm{PGE}_{2}$ overproduction and upregulation of its corresponding enzyme, COX-2, and this was significantly ameliorated by chrysoeriol treatment in a dose-dependent manner without cytotoxicity (Fig. 1A and B). Along with the effect on inflammatory signaling cascades, activation of NF- $\kappa \mathrm{B}$ and AP-1 is highly associated with the upregulation of inflammatory mediators, including iNOS and COX-2. Previous studies have also reported that both transcription factors NF- $\kappa$ B and AP-1 are critical regulators of inflammatory signaling pathways (30). $\mathrm{NF}-\kappa \mathrm{B}$ ubiquitously exists in the cytoplasm and consists of p50 and p65 subunits bound to I $\mathrm{B} \alpha$, while AP-1 resides as homo- or heterodimers with the c-jun and c-fos families (30). In response to LPS stimulation, $\mathrm{NF}-\kappa \mathrm{B}$ and AP-1 become active forms via phosphorylated $\mathrm{I} \kappa \mathrm{B} \alpha$, resulting in release from the $\mathrm{NF}-\kappa \mathrm{B}$ dimer and c-jun phosphorylation, respectively (30). In this study as shown in Fig. 2, the phosphorylated status of p65 and c-jun, each subunit of NF- $\mathrm{kB}$ and AP-1, was measured by western blot analysis, which was the phosphorylation of both transcription factors was significantly inhibited by chrysoeriol treatment in a dose-dependent manner. Moreover, activated MAPKs or PI3K/Akt can lead to a series of inflammatory signaling cascades via the regulation of NF- $\kappa$ B and AP-1 activation (31). As shown in Fig. 3A and B, chrysoeriol significantly inhibited PI3K/Akt and p38 phosphorylation levels, as well as slightly mitigated JNK activation, while ERK was not affected. In addition, selective inhibitors were applied to confirm the role of signaling molecules in LPS-stimulated inflammatory cascades. Each selective inhibitor of PI3K and p38 significantly attenuated COX-2 expression while JNK did not give any effect on COX-2 expression in this experiment. Cotreatment of selective inhibitors of these signaling molecules was the most potent inhibitory effect in LPS stimulated RAW 264.7 cells.

TLRs are a growing family of pattern recognition receptors (PRRs) which can stimulate innate immunity and inflammatory responses upon the interaction with numerous pathogen-associated molecular patterns including bacterial LPS, viral RNA, and flagellin (32). As a ligand for TLR4, LPS can bind to the extracellular domain of TLR4 and form intracellular adaptor molecules including MyD88 and Toll-interleukin 1 receptor domain-containing adaptor protein (TIRAP) $(17,32,33)$. Accelerated production of MyD88 can lead to the activation of NF- $\mathrm{B}, \mathrm{AP}-1, \mathrm{PI} 3 \mathrm{~K} / \mathrm{Akt}$, and MAPKs and the production of inflammatory mediators $(17,33)$. As shown in Fig. 4, chrysoeriol attenuated dose-dependently LPS initiated TLR4 and MyD88 activation in accordance with the inhibited NF- $\mathrm{B}$, AP-1, PI3K/Akt, and p38 MAPK expression levels, in LPS-stimulated RAW 264.7 cells.

In conclusion, the present results suggest that chrysoeriol signigicantly ameliorates LPS induced $\mathrm{PGE}_{2}$ production and COX-2 expression through the regulation of TLR4 and MyD88 mediated NF-кB, AP-1, PI3K/Akt, and MAPKs in RAW 264.7 cells.

\section{Acknowledgements}

Not applicable.

\section{Funding}

No funding was received.

\section{Availability of data and materials}

The datasets used and/or analyzed during the current study are available from the corresponding author on reasonable request.

\section{Author's contributions}

HSY and CMP made substantial contributions to the conceptualization and design of the study. HSY performed the experiments for data acquisition and conducted statistical analysis. HSY and CMP confirmed the authenticity of all the raw data. CMP interpreted the experimental results and wrote the manuscript; HSY and CMP revised the final manuscript. All authors read and approved the final manuscript.

\section{Ethics approval and consent to participate}

Not applicable.

\section{Patient consent for publication}

Not applicable.

\section{Competing interests}

The authors declare that they have no competing interests.

\section{References}

1. Kunnumakkara AB, Sailo BL, Banik K, Harsha C, Prasad S, Gupta SC, Bharti AC and Aggarwal BB: Chronic diseases, inflammation, and spices: How are they linked? J Transl Med 16: $14,2018$.

2. Kundu JK and Surh YJ: Inflammation: Gearing the journey to cancer. Mutat Res 659: 15-30, 2008.

3. Sukketsiri W, Tanasawet S, Moolsap F, Tantisira MH, Hutamekalin P and Tipmanee V: ECa 233 suppresses LPS-induced proinflammatory responses in macrophages via suppressing ERK1/2, p38 MAPK and akt pathways. Biol Pharm Bull 42: 1358-1365, 2019.

4. Park SA, Kim EH, Na HK and Surh YJ: KG-135 inhibits COX-2 expression by blocking the activation of JNK and AP-1 in phorbol ester-stimulated human breast epithelial cells. Ann NY Acad Sci 1095: 545-553, 2007.

5. Na HK and Surh YJ: Intracellular signaling network as a prime chemopreventive target of (-)-epigallocatechin gallate. Mol Nutr Food Res 50: 152-159, 2006.

6. Kim JH, Na HK, Pak YK, Lee YS, Lee SJ, Moon A and Surh YJ: Roles of ERK and p38 mitogen-activated protein kinases in phorbol ester-induced NF-kappaB activation and COX-2 expression in human breast epithelial cells. Chem Biol Interact 171: 133-141, 2008.

7. Kawamori T, Rao CV, Seibert K and Reddy BS: Chemopreventive activity of celecoxib, a specific cyclooxygenase-2 inhibitor, against colon carcinogenesis. Cancer Res 58: 409-412, 1998.

8. Choi CW, Jung HA, Kang SS and Choi JS: Antioxidant constituents and a new triterpenoid glycoside from flos lonicerae. Arch Pharm Res 30: 1-7, 2007.

9. Schinella GR, Giner RM, Recio MC, Mordujovich de Buschiazzo P, Rios JL and Manez S: Anti-inflammatory effects of South American Tanacetum vulgare. J Pharm Pharmacol 50: 1069-1074, 1998.

10. Abu Zarga M, Qauasmeh R, Sabri S, Munsoor M and Abdalla S: Chemical constituents of artemisia arborescens and the effect of the aqueous extract on rat isolated smooth muscle. Planta Med 61: 242-245, 1995.

11. Han LK, Sumiyoshi M, Zheng YN, Okuda H and Kimura Y: Anti-obesity action of salix matsudana leaves (Part 2). Isolation of anti-obesity effectors from polyphenol fractions of salix matsudana. Phytother Res 17: 1195-1198, 2003. 
12. Mishra B, Priyadarsini KI, Kumar MS, Unnikrishnan MK and Mohan H: Effect of O-glycosilation on the antioxidant activity and free radical reactions of a plant flavonoid, chrysoeriol. Bioorg Med Chem 11: 2677-2685, 2003.

13. Choi DY, Lee JY, Kim MR, Woo ER, Kim YG and Kang KW: Chrysoeriol potently inhibits the induction of nitric oxide synthase by blocking AP-1 activation. J Biomed Sci 12: 949-959, 2005.

14. Kim JH, Cho YH, Park SM, Lee KE, Lee JJ, Lee BC, Pyo HB, Song KS, Park HD and Yun YP: Antioxidants and inhibitor of matrix metalloproteinase-1 expression from leaves of Zostera marina L. Arch Pharm Res 27: 177-183, 2004.

15. Park CM and Song YS: Luteolin and luteolin-7-O-glucoside inhibit lipopolysaccharide-induced inflammatory responses through modulation of NF-kB/AP-1/PI3K-Akt signaling cascades in RAW 264.7 cells. Nutr Res Pract 7: 423-429, 2013.

16. Bak MJ, Truong VL, Kang HS, Jun M and Jeong WS Anti-inflammatory effect of procyanidins from wild grape (Vitis amurensis) seeds in LPS-induced RAW 264.7 cells. Oxid Med Cell Longev 2013: 409321, 2013.

17. Chun J, Choi RJ, Khan S, Lee DS, Kim YC, Nam YJ, Lee DU and Kim YS: Alantolactone suppresses inducible nitric oxide synthase and cyclooxygenase-2 expression by down-regulating NF-kB, MAPK and AP-1 via the MyD88 signaling pathway in LPS-activated RAW 264.7 cells. Int Immunopharmacol 14: 375-383, 2012

18. Jeong D, Dong GZ, Lee HJ and Ryu JH: Anti-inflammatory compounds from atractylodes macrocephala. Molecules 24 : $1859,2019$.

19. Xie WL, Chipman JG, Robertson DL, Erikson RL and Simmons DL: Expression of a mitogen-responsive gene encoding prostaglandin synthase is regulated by mRNA splicing. Proc Natl Acad Sci USA 88: 2692-2696, 1991.

20. Yang Y, Zhou X, Xiao M, Hong Z, Gong Q, Jiang L and Zhou J: Discovery of chrysoeriol, a PI3K-AKT-mTOR pathway inhibitor with potent antitumor activity against human multiple myeloma cells in vitro. J Huazhong Univ Sci Technolog Med Sci 30: 734-740, 2010

21. Medzhitov R: Origin and physiological roles of inflammation. Nature 454: 428-435, 2008.

22. Tabas I and Glass CK: Anti-inflammatory therapy in chronic disease: Challenges and opportunities. Science 339: 166-172, 2013.
23. Qian C, Liu J and Cao X: Innate signaling in the inflammatory immune disorders. Cytokine Growth Factor Rev 25: 731-738, 2014.

24. Schindler SM, Little JP and Klegeris A: Microparticles: A new perspective in central nervous system disorders. Biomed Res Int 2014: 756327, 2014.

25. Van Dyke TE and van Winkelhoff AJ: Infection and inflammatory mechanisms. J Periodontol 84 (Suppl 4): S1-S7, 2013.

26. Meng F and Lowell CA: Lipopolysaccharide (LPS)-induced macrophage activation and signal transduction in the absence of Src-family kinases Hck, Fgr, and Lyn. J Exp Med 185: 1661-1670, 1997.

27. Roy A, Srivastava M, Saqib U, Liu D, Faisal SM, Sugathan S, Bishnoi S and Baig MS: Potential therapeutic targets for inflammation in toll-like receptor 4 (TLR4)-mediated signaling pathways. Int Immunopharmacol 40: 79-89, 2016.

28. Hu W, Wu L, Qiang Q, Ji L, Wang X, Luo H, Wu H, Jiang Y, Wang $\mathrm{G}$ and Shen T: The dichloromethane fraction from Mahonia bealei (Fort.) Carr. leaves exerts an anti-inflammatory effect both in vitro and in vivo. J Ethnopharmacol 188: 134-143, 2016.

29. Wang J, Kang YX, Pan W, Lei W, Feng B and Wang XJ: Enhancement of anti-inflammatory activity of curcumin using phosphatidylserine-containing nanoparticles in cultured macrophages. Int J Mol Sci 17: 969, 2016.

30. Surh YJ: Cancer chemoprevention with dietary phytochemicals Nat Rev Cancer 3: 768-780, 2003.

31. Endale M, Park SC, Kim S, Kim SH, Yang Y, Cho JY and Rhee MH: Quercetin disrupts tyrosine-phosphorylated phosphatidylinositol 3-kinase and myeloid differentiation factor-88 association, and inhibits MAPK/AP-1 and IKK/NF-kB-induced inflammatory mediators production in RAW 264.7 cells. Immunobiology 218: 1452-1467, 2013.

32. Chen K, Huang J, Gong W, Iribarren P, Dunlop NM and Wang JM: Toll-like receptors in inflammation, infection and cancer. Int Immunopharmacol 7: 1271-1285, 2007.

33. Dauphinee SM and Karsan A: Lipopolysaccharide signaling in endothelial cells. Lab Invest 86: 9-22, 2006. 